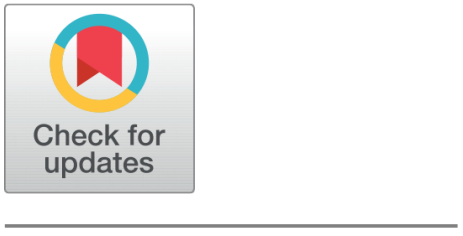

open ACCESS

Received: 01.04.2021

Accepted: 24.05.2021

Published: 12.07 .2021

Citation: Padinhattayil S, Rai KS (2021) PVA/GO-ZnO hybrid nanocomposites: Synthesis, Analysis and Applications. Indian Journal of Science and Technology 14(23): 1982-1992. https://doi.org/ 10.17485/IJST/v14i23.236

* Corresponding author.

sanoop.home@gmail.com

Funding: None

Competing Interests: None

Copyright: @ 2021 Padinhattayil \& Rai. This is an open access article distributed under the terms of the Creative Commons Attribution License, which permits unrestricted use, distribution, and reproduction in any medium, provided the original author and source are credited.

Published By Indian Society for Education and Environment (iSee)

ISSN

Print: 0974-6846

Electronic: 0974-5645

\section{PVA/GO-ZnO hybrid nanocomposites: Synthesis, Analysis and Applications}

\author{
Sanoop Padinhattayil ${ }^{1 *}$, K Sheshappa Rai ${ }^{1}$ \\ 1 Research Scholar, Department of Post-graduate Studies and Research in Polymer Science, \\ University of Mysore, Sir M Visvesvaraya Post-graduate Centre, Tubinakere, Mandya, 571 402, \\ Karnataka, India
}

\section{Abstract}

Objective: To prepare Poly vinyl alcohol (PVA) nanocomposites with novel GO-ZnO hybrid Nano filler and test its efficacy and applications. Methods: GO-ZnO hybrid nanocomposite filler was synthesized by modified hummer's method. Polymer nanocomposite films were prepared by solution casting method. Synthesis of GO-ZnO hybrid procedure is explained in detail. ZnO with average size of $26.74 \mathrm{~nm}$ were equally anchored on the $\mathrm{GO}$ surface. The properties of the PVA film and the PVA/Graphene Oxide/Zinc oxide composite film were analyzed by electrical, thermo mechanical \& spectroscopic analysis. Findings: The results showed that the incorporation of graphene oxide and zinc oxide into the PVA matrix resulted in enhanced mechanical properties and thermal stability of PVA composite films. The tensile strength increases from 3.84 MPa of neat PVA to $10.48 \mathrm{MPa}$ of PVA-0.05\% GO-ZnO and Young's modulus increases from 104.407 to $106.765 \mathrm{MPa}$. The elongation behavior of the Polymer is increased by the addition of Nano filler and it varies from $15.94 \mathrm{Mpa}$ of pure PVA polymer film to $239.52 \mathrm{MPa}$ of $1 \%$ loading -GO-ZnO hybrid filler. Such an enormous improvement in the mechanical properties at such low stacking of GO-ZnO can be credited to a property of GO-ZnO hybrid nano-filler. DC conductivity studies were also under taken on the samples and the conductivity was found to be $2.895 \times 10-6 \mathrm{~S} \cdot \mathrm{cm}-1$ for $5 \%$ loading of nano particle while pure PVA is observed as $9.952 \times 10-8$.

Keywords: GOZnO; Hybrid; filler; nanocomposite; PVA; Electrical Conductivity

\section{Introduction}

Polymer-nanocomposites played a significant role in the field of polymer nanotechnology for different innovative/modern applications. The properties of polymer nanocomposites display totally unexpected nature in comparison to the polymeric matrix. This is because of the excellent capacities of the nano-sized fillers and the expanded surface area at the interface. The nanoparticles properties, for example, molecule size, molecule size circulation, scattering state, geometric shape and surface properties are the other notable components, which adjust the material properties of the nanocomposites. ${ }^{(1,2)}$ Depending upon the chemical nature of the fusing nano-particles and the manner by 
which they collaborate with the polymer matrix, the physical properties of polymer nanocomposites differs at various degrees. ${ }^{(3)}$ The exceptional properties of polymer nanocomposites emerge from association of its phases at the interfaces. Graphene based materials have attracted tremendous amount of interest in many areas of science and technology due to the excellent properties of graphene ${ }^{(4-11)}$, as determined by the theoretical and experimental results, individual graphene sheets exhibit extremely high value of Young's modulus $(\sim 1000 \mathrm{Gpa})$, fracture strength $(\sim 125 \mathrm{Gpa})^{(12)}$, elastic modulus $(\sim 0.25 \mathrm{Tpa}){ }^{(13)}$ The structure of Graphene Oxide is often assumed to be a graphene sheet bonded to oxygen in the form of carbonyl, hydroxyl and epoxy groups $^{(14)}$.

To confine Graphene Oxide (GO) from agglomerating and restacking, functionalization of GO has been performed. The three fundamental classes of GO functionalization will be by organic sources, functionalization of macromolecules, and functionalization by nanoparticles $(\mathrm{Nps})^{(12)}$ Zinc oxide $(\mathrm{ZnO})$ is a multifunctional material, because of its remarkable physical and chemical properties, including chemical stability, no toxicity, and cost efficiency ${ }^{(13,14)}$. Apart from its capacity as an UVblocking agent ${ }^{(15)}$, the antibacterial and antifungal properties of $\mathrm{ZnO}$ make it valuable for surface covering, materials, and plastics applications ${ }^{(16)}$. When combined with different NPs, for example, titanium oxide and alumina, $\mathrm{ZnO}$ can undoubtedly scatter because of its steady surface ${ }^{(17)}$. An easy course was created to frame graphene oxide-zinc oxide nanohybrids $(\mathrm{GO} / \mathrm{ZnO})$ and blending the special structure and properties of GO sheets with the promising properties of ZnO Nano particles will undoubtedly present a novel and practical change in the polymer matrix ${ }^{(18)}$.

Poly (vinyl Alcohol) (PVA) is a transparent hydrophilic polymer that has been generally utilized in industry for quite a while ${ }^{(19-22)}$. PVA is known to have solid oxygen barrier properties because of the solid hydrogen bonding of the hydroxyl $(\mathrm{OH})$ groups in PVA, just as better rigidity, elongation, and abrasion resistance than general engineered materials.PVA as of now utilized in different fields, for example, fibers, films, adhesives, surface treatment chemicals, and coating materials ${ }^{(23-25)}$. Because of the hydrophilicity of PVA, its mechanical properties and electrical properties change significantly relying upon the outer humidity and its solubility, thickness ${ }^{(26-28)}$. PVA is a water-soluble polymer broadly explored as a host for various types of Nano fillers ${ }^{(20-25,29)}$. These observations recommend that the presentation of Nanosized particles into PVA do modify its properties and works as excellent biopolymer. Diversification in nano filler classes with multifunctionalities, increased structural complexity, uniform dispersions, in-depth understanding of interfacial interactions, and better control over synthesis steps with controlled morphologies are all possible issues in the production of PVA nanocomposites in the near future. These issues necessitate the improvement of experimental methods, the enhancement of a systemic method, and the acquisition of advanced specific information. As a result, substantial progress in promoting significant innovation in industry and small medium enterprises could be possible. It is hoped that the researchers would be able to resolve the problems, and that PVA nanocomposites will have a bright future.

In this study, PVA films were prepared using GO/ZnO hybrid nano filler by a solution casting method. The hybrid Nano filler with nano size was introduced by wet chemical synthesis route by using $20.74 \mathrm{~nm} \mathrm{ZnO}$ nanoparticle in our lab and the properties were explored ${ }^{(18)}$. The nano size of GO-ZnO is calculated by using scherrer's formula and found as $50.94 \mathrm{~nm}^{(18)}$. The thermal properties, morphology, optical study, and electrical conductivity of PVA containing the hybrid Nano fillers were investigated.

\section{Materials and Methods}

\subsection{Materials}

Poly vinyl alcohol (PVA) (MW = 1, 25,000), AR grade from Merck India Ltd, India, GO-ZnO Hybrid nano fillers are synthesized in and the detailed procedure is explained in the experimental procedure and Distilled water is used for all experiments in the lab

\subsection{Synthesis of GO/ZnO hybrid}

The GO/ZnO hybrid nanocomposite was synthesized by simple wet chemical method. In this method, Graphene oxide was dispersed in ethanol (2:1 wt/ vol ratio) and the solution mixture was sonicated for $1 \mathrm{hr}$ at room temperature. Afterward $\mathrm{ZnC}_{4} \mathrm{H}_{6} \mathrm{O}_{4} .2 \mathrm{H}_{2} \mathrm{O}(0.880 \mathrm{~g})$ was dissolved in to the mixer. The ${ }_{p} \mathrm{H}$ of the solution was adjusted to 10 by adding $1 \mathrm{M} \mathrm{NaOH}$ solution and stirred for $30 \mathrm{~min}$. the mixture was refluxed at $140^{\circ} \mathrm{C}$ under inert atmosphere for $24 \mathrm{hrs}$. The prepared hybrid nanocomposites were centrifuged and washed with ethanol and distilled water for several times. Product was dried under vacuum oven for 6 hours to get fine powder of $\mathrm{GO} / \mathrm{ZnO}$ hybrid nanocomposites 


\subsection{Preparation of composite films}

The solution casting system was utilized for the manufacture of PVA/GO-ZnO polymer nanocomposite films. 4\% PVA solution was prepared by dissolving PVA in double distilled water $(4 \mathrm{wt} \%)$ at $70-80^{\circ} \mathrm{C}$. After that to the uniform solution of PVA, GO-ZnO nanoparticles were added at various proportions of 1,3 and $5 \mathrm{wt} \%$. The mixture was kept for blending around 24 h. Ultra sonicator was utilized with $10 \mathrm{~Hz}$ for 15-30 min to homogenize the mixture. The homogeneous solution was poured in polypropylene dishes and kept in hot air oven at $65-70^{\circ} \mathrm{C}$ for 24 hours for the formation of thin film. A fine nanocomposite polymer film was observed. The film was kept in desiccators for preservation for further studies.

\subsection{Structural characterization}

The X-Ray Diffractometer (XRD) for the PVA and composite film structure of the PVA/GO-ZnO nanocomposite was done in a Bruker diffractometer (model $8600 \mathrm{USA}$ ) with $\mathrm{Cu}$ Ka radiation $(\mathrm{k}=0.15406 \mathrm{~nm})$. The voltage was set at $40 \mathrm{kV}$ at a scanning rate of $2^{\circ} / \mathrm{min}$ from $10^{\circ}$ to $80^{\circ}(2 \theta)$ angles.

FTIR offers quantitative and subjective investigation of organic compounds. Fourier Transform Infrared Spectroscopy (FTIR) distinguishes chemical bonds in a compound by creating an infrared absorption range. FTIR spectra of pure PVA and distinctive nanocomposite films were acquired a spectrum in the range from 4000 to $550 \mathrm{~cm}^{-1}$.

\subsection{Thermal and Mechanical characterization}

Thermal properties of the PVA/GO-ZnO nanocomposite films were drawn by Perkin Elmer DSC/TGA instrument (model exstar Japan). The nanocomposite films were warmed from $30^{\circ} \mathrm{C} / \mathrm{min}$ under $\mathrm{N}_{2}$ medium.

\subsection{UV-VIS analysis}

Optical analysis carried for all the samples by UV-1800 (Shimadzu-Japan) spectrophotometer. The mathematical relationship of Tauc was used to calculate the band gap energy (Eg) of PVA and PVA/GO-ZnO nanocomposite films. ${ }^{(30)}$ Results are tabulated in the Results and Discussion section.

\subsection{Electrical conductivity analysis}

A significant property of many materials is sheet resistance (or surface resistivity), quantifying the ability of charges to move across uniform thin films. The four-probe technique is the most appropriate method used for monitoring sheet resistance. In order to make electrical contact with the material, this technique involves using four equally spaced, co-linear probes known as a four-point probe ${ }^{(31,32)}$. Most widely available four-point probes have sharp needles as probes. The four-probe approach works by penetrating the substance with four equal-spaced, co-linear probes between the outer two probes ( 1 and 4), DC current is applied and a voltage drop is determined between the inner two probes ( 2 and 3$)$. It is then possible to calculate the sheet resistance using the following equation:

$$
\rho=2 \pi s\left(\frac{V}{I}\right)
$$

Where $\mathrm{V}$ is the potential difference in volts between internal probes, ' $\mathrm{I}$ ' is the current through the outer pair of ampere probes and 'S' the Spacing between meter probes ${ }^{(33-36)}$.

\section{Results and Discussion}

\subsection{XRD characterization}

XRD is a significant instrument for describing the delamination and scattering of GO-ZnO hybrid fillers in the nanocomposites. Figure 1 shows the XRD pattern of neat PVA, and PVA/GO-ZnO nanocomposite films. In comparison to pure PVA, the XRD patterns of PVA/GO-ZnO nanocomposite display the characteristic peaks of $\mathrm{ZnO}$ nanoparticles at $2 \theta=31.76^{\circ}, 34.36^{\circ}, 36.24^{\circ}$, and $47.54^{\circ}$, which correspond to the reflection planes of ZnO's hexagonal wurtzite crystal structure. These additional peaks are the same as those found in $\mathrm{ZnO}$ Nanoparticles ${ }^{(22)}$.

Furthermore, the $\left(\begin{array}{lll}1 & 0 & 1\end{array}\right)$ diffraction peak of PVA crystals at $2 \theta=19.48^{\circ}$ is a very intense peak from the composites after adding the $\mathrm{ZnO}$ NPs and GO. However, its location drops to $19.2^{\circ}$, its strength drops, and the crystallisation peak broadens, indicating that there is some contact between the three compounds which is logical with Yang, Shuai, et al ${ }^{(37,38)}$. All the new 
existing diffraction peaks are related with the diffraction peaks of GO-ZnO hybrid nanoparticles. It further confirms the proper miscibility of PVA with GO-ZnO hybrid nanoparticles.
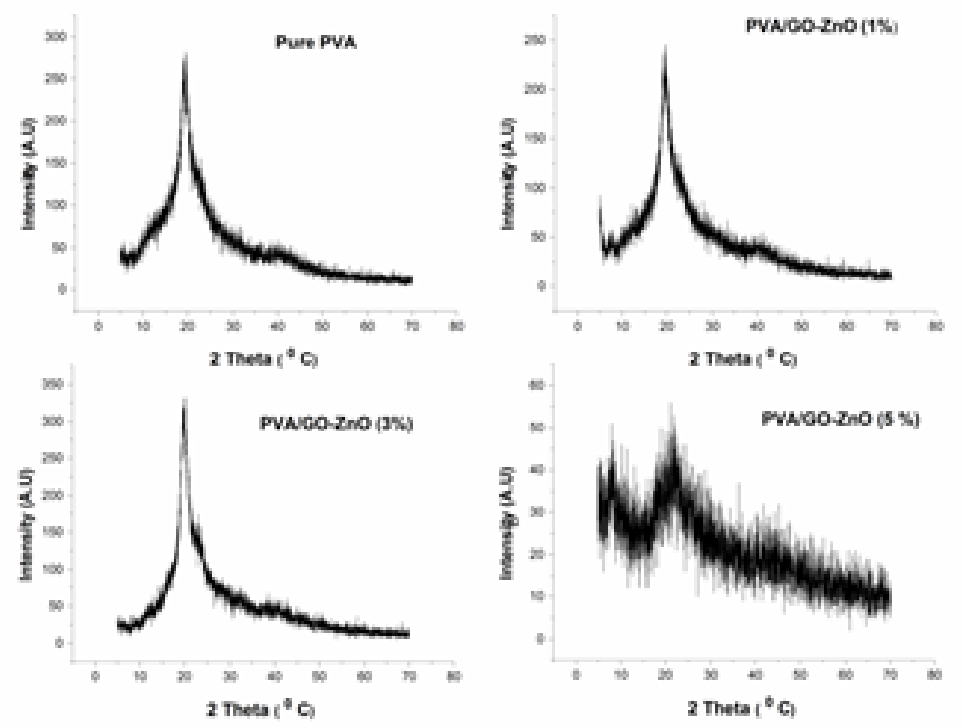

Fig 1. XRD Chromatogram Spectra of PVA nanocomposite films with different weight percentage of GO-ZnO filler.

\subsection{FTIR characterization}

Figure 2 shows the FTIR spectra of PVA and PVA/GO-ZnO nanocomposites. The peaks related with the structure of the PVA are represented here. A wide band at $3290 \mathrm{~cm}^{-1}$ corresponds to hydroxyl stretching $(\mathrm{O}-\mathrm{H})$ of the introduced PVA matrix. A peak at $2941 \mathrm{~cm}^{-1}$ is attributed to $\mathrm{C}-\mathrm{H}$ asymmetric vibration ${ }^{(39)}$. A tight peak at $1715 \mathrm{~cm}^{-1}$ is identified with the carbonyl extension $(\mathrm{C}=\mathrm{O})$ of PVA acetyl group. A top at $1247 \mathrm{~cm}^{-1}$ is identified with the $\mathrm{C}-\mathrm{O}-\mathrm{C}$ stretching of the host matrix. The top at $833 \mathrm{~cm}^{-1}$ relates with the $-\mathrm{CH} 2$ extending vibration of PVA polymer. The above represented peaks are almost present for all the PVA/GO-ZnO hybrid nanocomposite films (Figure 2) apart from this, the peaks of $\mathrm{C}=\mathrm{O}$ and $\mathrm{C}-\mathrm{O}-\mathrm{C}$ extending vibrations are surprisingly moved from their real positions when compare with pure PVA positions. This strongly demonstrates the actual collaboration between nano-sized hybrid GO-ZnO filler and the PVA matrix

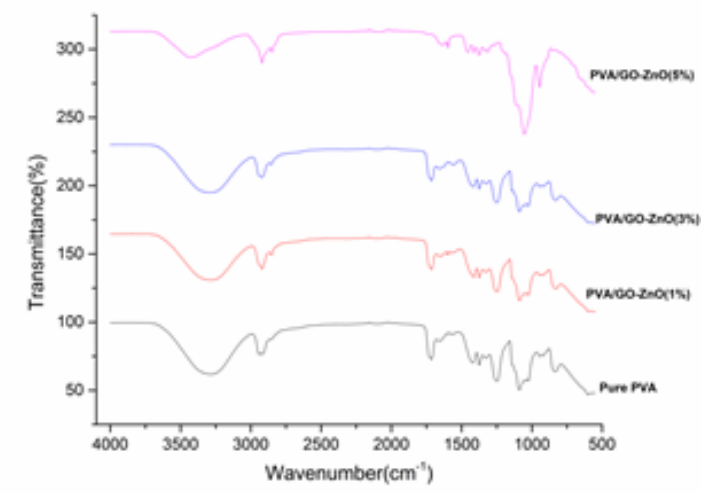

Fig 2. FTIR analysis Spectra of PVA nanocomposite films with different weight percentage of GO-ZnO filler 


\subsection{Morphological characterization}

Morphological images of PVA/GO-ZnO composites was analyzed by SEM. Figure 3 illustrates SEM micrographs for the fracture surfaces of neat PVA and PVA/GO-ZnO nanocomposite. The neat PVA fracture surface appears as smooth surface. However, the fracture surface morphology is different after the GO- $\mathrm{ZnO}$ hybrid is incorporated
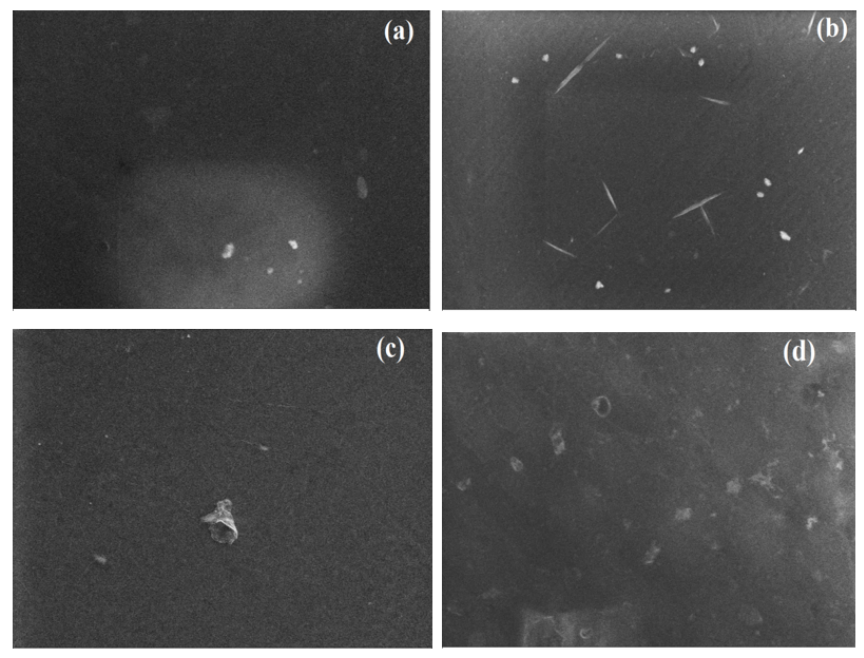

Fig 3. SEM images of (a) Pure PVA, (b) PVA/GO-ZnO (1 wt \%), (c) PVA/GO-ZnO (3 wt \%) and (d) PVA/GO-ZnO (5 wt \%)

Figure 3(a) explains about the SEM micrograph of perfect PVA film. It appears exceptionally dark without any voids. It confirms that the film has no air bubbles. The surface morphology of the nano-sized GO-ZnO hybrid Nano filler incorporated PVA nanocomposite film was additionally examined to understand the actual connection of nano-sized GO-ZnO with the polymer matrix. The SEM micrographs of polymer nanocomposite films are shown in Figure 3(b-d). At lower rate stacking of $\mathrm{GO}-\mathrm{ZnO}(1 \%)$ the PVA is nicely mixed and distributed with nanoparticles, at higher rate of stacking GO-ZnO nanoparticles, it was seen that agglomeration rate is also increasing because of the high surface energy of GO-ZnO Nanoparticles. ${ }^{(40)}$ All the polymer nanocomposite films were prepared with no air bubbles which could be seen from the SEM images.

\subsection{Thermal characterization}

The Thermal dependability of PVA/GO-ZnO nanocomposites can be dissected from TGA-DSC methods. The TGA thermograms of the readied PVA/GO-ZnO composite films are appeared in Figure 4 . The initial step of debasement around $100^{\circ} \mathrm{C}$ shows the loss of moisture and evaporation of trapped solvent. The second step of degradation shows up in the temperature range between $100^{\circ} \mathrm{C}-200^{\circ} \mathrm{C}$ demonstrates the thermal stage change at a beginning phase in nanocomposite films. The third degradation between $200^{\circ} \mathrm{C}-300^{\circ} \mathrm{C}$ demonstrates the disintegration of composite films identified with side chain or functional group disentanglement. The fourth step of over $400^{\circ} \mathrm{C}$ corresponds to the complete degradation curve of the polymer.

The percentage of weight loss of and PVA/GO-ZnO hybrid nanocomposite films at various temperatures is given in Table 1. The TGA thermograms of PVA and PVA/GO-ZnO polymer nanocomposite films (Figure 3) show the increase in percentage of GO-ZnO filler to PVA matrix extrapolating the thermal stability of the polymer. At $300^{\circ} \mathrm{C}$ the level of weight reduction of PVA pure film is $25.45 \%$ and it is diminishing to $16.77 \%$ when it is added with $5 \%$ of GO-ZnO. This also consolidates the expansion in thermal stability of PVA film after the addition of GO-ZnO.

The DSC behavior of the PVA and PVA/GO-ZnO hybrid nanocomposite films are shown in Figure 5 . The glass transition temperature $\left(\mathrm{T}_{g}\right)$ of the PVA pure film is $103.79^{\circ} \mathrm{C}$. As the percentage of Hybrid nano filler increases the $\mathrm{T}_{g}$ of the film also increases. For $1 \%$ \& $2 \%(\mathrm{w} / \mathrm{v})$ loading of Hybrid nano filler the $\mathrm{T}_{g}$ values are $104.91^{\circ} \mathrm{C}$ and $105.1^{\circ} \mathrm{C}$ respectively, but there is no remarkable increase in glass transition temperature is observed with $5 \%(\mathrm{w} / \mathrm{v})$ of filler charge. Similarly $\mathrm{T}_{g}$ and the melting temperature (Tm) of the PVA/GO-ZnO polymer nanocomposite films increased with increase in incorporation of hybrid filler in to the PVA matrix. The pure PVA start melting at $186.46^{\circ} \mathrm{C}$ and the melting temperature increases to $187.39^{\circ} \mathrm{C} \& 188.29^{\circ} \mathrm{C}$ by the addition of hybrid nano filler. This indicates that the GO-ZnO hybrid nano filler played an important role in boosting the thermal properties of the polymer. 


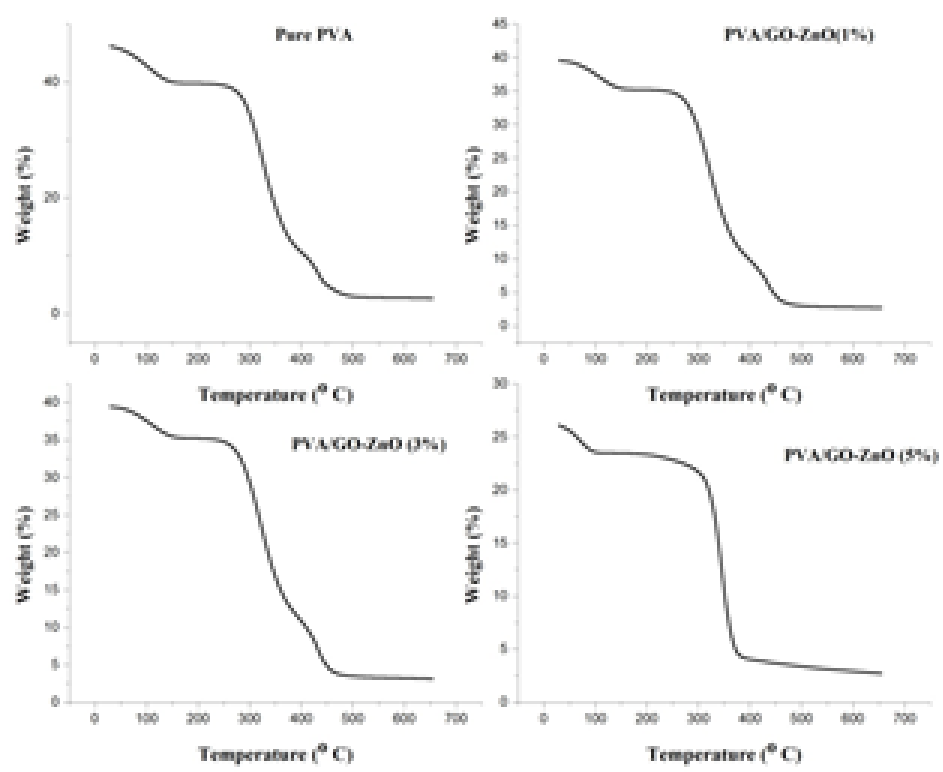

Fig 4. TGA analysis Spectra of PVA nanocomposite films with different weight percentage of GO-ZnO filler

Table 1. Percentage of weight loss of PVA/GO-ZnO hybrid nanocomposites at different temperatures

\begin{tabular}{|c|c|c|c|c|c|c|c|}
\hline \multirow{2}{*}{ Sample code } & \multicolumn{7}{|c|}{ Percentage of weight loss at various temperature } \\
\hline & $100^{\circ} \mathrm{C}$ & $200^{\circ} \mathrm{C}$ & $300^{\circ} \mathrm{C}$ & $400^{\circ} \mathrm{C}$ & $500^{\circ} \mathrm{C}$ & $600^{\circ} \mathrm{C}$ & Residue \\
\hline Pure PVA & 7.3 & 13.92 & 25.45 & 77.01 & 93.52 & 94.10 & 5.9 \\
\hline PVA/ GO-ZnO (1\%) & 5.12 & 11.04 & 27.47 & 75.60 & 92.41 & 92.9 & 7.1 \\
\hline PVA/ GO-ZnO (3\%) & 4.74 & 10.75 & 26.28 & 72.44 & 91.9 & 91.79 & 8.21 \\
\hline PVA/ GO-ZnO (5\%) & 9.60 & 12.29 & 16.77 & 84.42 & 87.07 & 90.75 & 9.25 \\
\hline
\end{tabular}

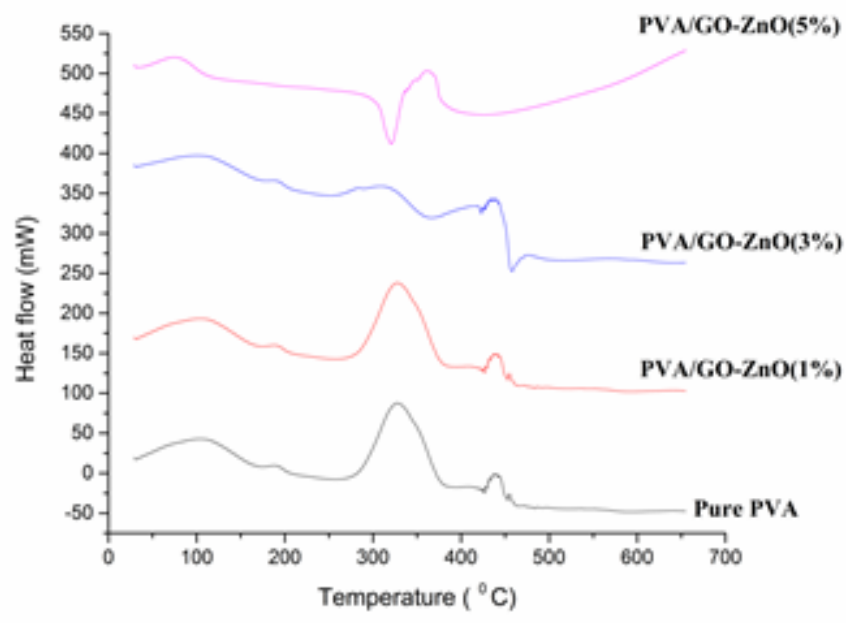

Fig 5. DSC analysis Spectra of PVA nanocomposite films with different weight percentage of GO-ZnO filler 


\subsection{Mechanical characterization}

The homogeneity of composites and the solid interfacial interaction between the hybrid nano film and the polymer network supposed to have significant impact on the mechanical properties. Figure 6 shows the mechanical properties of PVA/GO-ZnO hybrid polymer nano-composites:

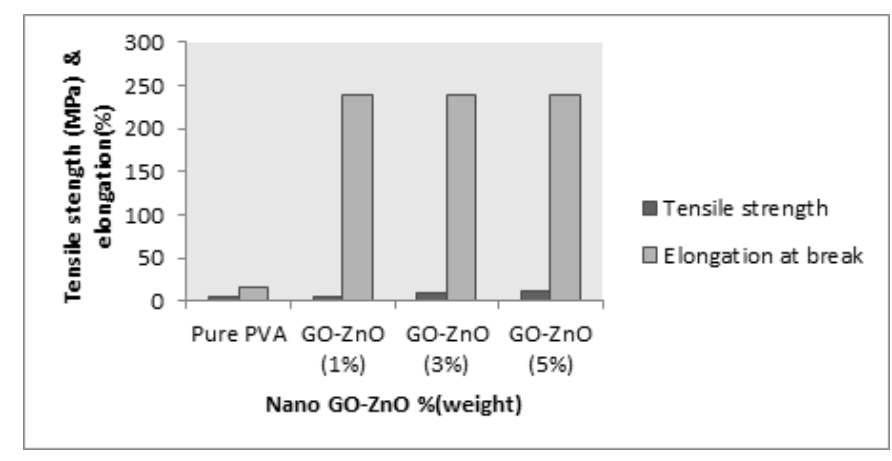

Fig 6. mechanical properties of PVA and Nanocomposites with different weight loadingof GO-ZnO nano particle

The subtle differences of the mechanical properties are tabulated in Table 2. As observed, both the tensile strength and Young's modulus evidently displayed an increasing pattern with the increase in GO-ZnO content. Zhang, Rong, et al ${ }^{(41)}$ explained the PVA membrane with a GO-to-ZnO Nanoparticle hybrid material with mass ratio of 3:7 exhibited the highest elongation at break at $177.14 \% \pm 7.17 \%$ where as in the present study the elongation behavior of the Polymer is increased by the addition of hybrid Nano filler and it varies from 15.94 Mpa of pure PVA polymer film to $239.52 \mathrm{MPa}$ to $1 \%$ loading of GO-ZnO hybrid filler. The elongation nature is observed to be constant or not much differed after reaching the extreme point (more than 60\%) from the pure PVA polymer film to Nano filler polymer matrix. The reduced crystallinity leads to increase in the elongation and tensile strength of the polymer. The GO/ZnO Nanocomposite incorporated PVA with a ratio of 3:7 shows the tensile strength of $11.92 \pm$ $0.58 \mathrm{Mpa}^{(41)}$ which is almost near value than PVA/GO-ZnO value $10.48 \mathrm{Mpa}$ as with $5 \%$ loading of GO-ZnO nanocomposites in the in the current work. However, the tensile strength increases from 3.84 MPa of neat PVA to 10.48 MPa of PVA-0.05\% GO-ZnO and Young's modulus increases from 104.407 to $106.765 \mathrm{MPa}$. It is clear indication of change in individual property of $\mathrm{ZnO}$ and such enormous improvement in the mechanical properties at such low stacking of GO-ZnO can be credited to a property of GO-ZnO hybrid nano-filler and the uniform distribution of the GO-ZnO fillers through the polymer matrix. The slight diminishing in Tensile strength and Young's modulus of PVA- $0.01 \%$ GO-ZnO is ascribed to the poor regularity at this loading percentage

Table 2. Mechanical properties of Pure PVA and PVA/GO-ZnO nanocomposites

\begin{tabular}{llll}
\hline Sample code & Young's modulus (MPa) & Elongation at break (\%) & Tensile strength (MPa) \\
\hline Pure PVA & 104.407 & 15.94 & 3.84 \\
PVA/ GO-ZnO (1\%) & 61.307 & 239.52 & 5.82 \\
PVA/ GO-ZnO (3\%) & 104.839 & 239.4 & 9.75 \\
PVA/ GO-ZnO (5\%) & 106.765 & 239.78 & 10.48 \\
\hline
\end{tabular}

\subsection{Optical characterization}

A change in the molecular structure of the polymer causes change within the optical band gap. The optical band gap information is accessible from the UV-Visible spectrum absorption edge of the PVA/GO-ZnO NP nanocomposite films, as shown in Figure 7 . The optical band gap in the plots of $(\alpha \mathrm{h} v)^{\frac{1}{2}}$ versus (hv) was determined by extrapolating straight regions to the (hv) axis, where $\alpha$ is the absorption coefficient and $\mathrm{h} v$ is the energy of the light photon incident.

Table 3 lists the obtained optical band gap energies of the nanocomposite films. The optical band gap of Pure PVA observed as $4.51 \mathrm{eV}$ and after the addition of $1 \%$ of GO-ZnO It is shifted as $4.32 \mathrm{eV}$, also it is evident from Table 3 that with the rise in Nano particle incorporation band gap energies decreases. The cross linking which resulted because of addition of GO-ZnO nanoparticles in the amorphous region of the polymer matrix would induce a shift in the polymer network crystal structure. 


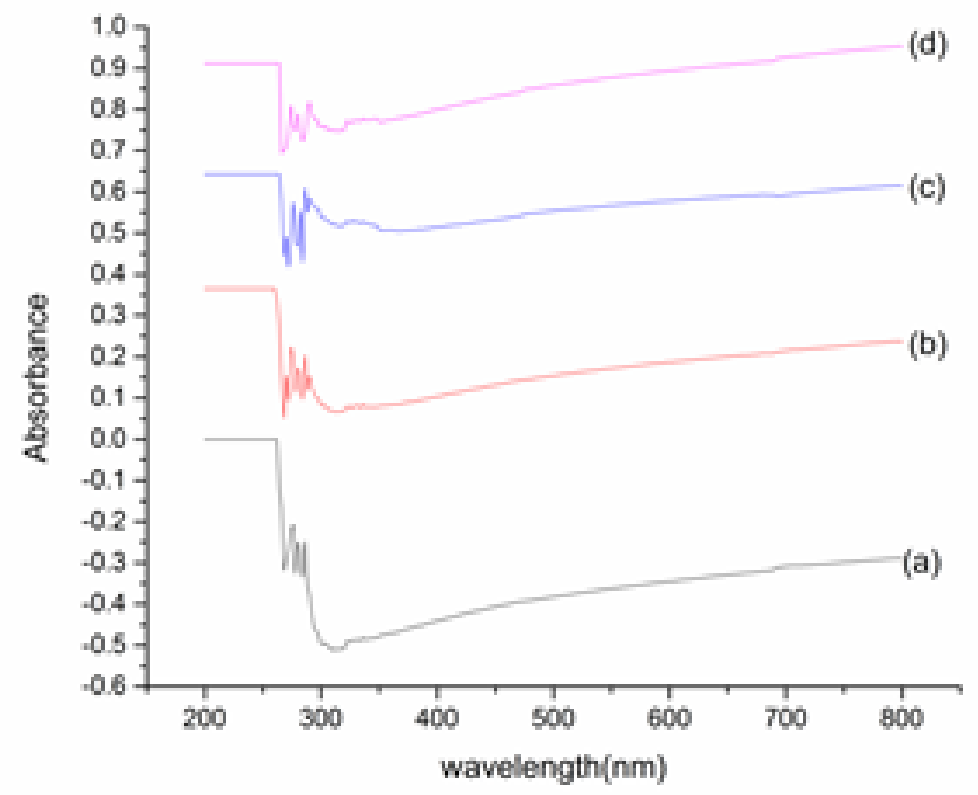

Fig 7. UV-VIS spectra of (a) Pure PVA, (b) PVA/GO-ZnO (1 wt \%), (c) PVA/GO-ZnO (3 wt \%) and (d) PVA/GO-ZnO (5 wt \%)

The decrease in the band gap energy indicates that the polymer become more conductive in nature as the nanoparticle dosage increases.

Table 3. Optical band gap energies of Pure PVA and PVA/GO-ZnO nanocomposites

\begin{tabular}{ll}
\hline Sample code & Band gap (eV) \\
\hline Pure PVA & 4.51 \\
PVA/ GO-ZnO (1\%) & 4.32 \\
PVA/ GO-ZnO (3\%) & 4.26 \\
PVA/ GO-ZnO (5\%) & 4.24 \\
\hline
\end{tabular}

\subsection{Electrical characterization}

Using a four-probe technique, the DC conductivity of PVA and PVA/GO-ZnO nanocomposites was studied at room temperature. The plot of GO-ZnO NPs electrical conductivity versus weight percent loading is shown in Figure 8 . After incorporating GO-ZnO NPs, there is a steady improvement in the conductivity of PVA. The conductivity of the PVA with $\mathrm{GO}$ and $\mathrm{ZnO}$ loading were shown a slight increases when compare with the Pure PVA and

After evaluating Table 4 and the figure it was concluded that the integrated GO-ZnO Nanoparticles created the conducting paths within the PVA matrix through a newly implemented charge transfer complex which results in an increase in the conductivity.

Table 4. Electrical conductivity properties of Pure PVA and PVA/GO-ZnO nanocomposites

\begin{tabular}{ll}
\hline Sample Code & Conductivity $\left(\mathbf{s ~ c m}^{-\mathbf{1}}\right)$ \\
\hline PVA & $9.952 \times 10^{-8}$ \\
PVA/GO-ZnO (1\%) & $5.983 \times 10^{-7}$ \\
PVA/GO-ZnO (3\%) & $1.220 \times 10^{-6}$ \\
PVA/GO-ZnO (5\%) & $2.895 \times 10^{-6}$ \\
\hline
\end{tabular}




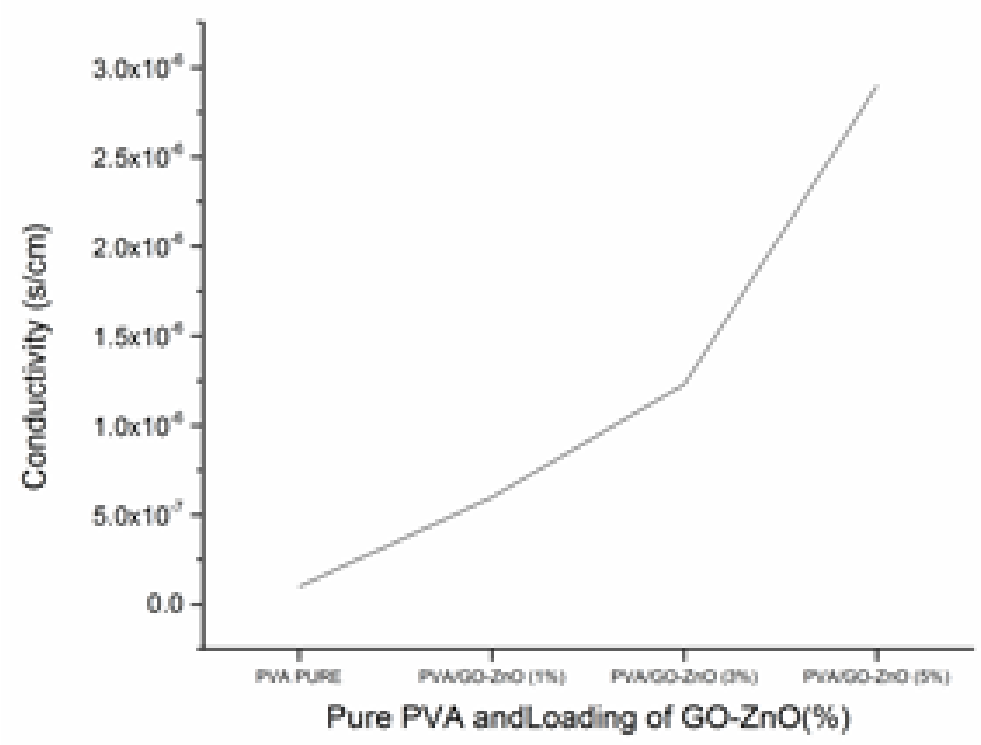

Fig 8. Electrical conductivity plot of PVA nanocomposite films with different weight percentage of GO-ZnO filler

\section{Conclusion}

The biopolymer PVA/GO-ZnO hybrid nanocomposites were effectively prepared by solution casting method. The XRD tests, SEM and Fourier transform infrared spectrometry confirmed the presence of GO-ZnO in PVA matrix. The Nano film basic investigation revealed that GO-ZnO hybrid nanoparticles were homogenously dispersed in the polymer bed with great interaction in polymer strings. Also, it was observed that by adding GO-ZnO hybrid Nano filler to the PVA polymer, the mechanical properties of nanocomposites were improved. Mechanical properties, for example, Tensile strength, Young's modulus and extension at break of PVA properties were upgraded with the loading of GO-ZnO. The tensile strength of PVAGO-ZnO (5\%) percent increases from 3.84 MPa to 10.48 MPa, and the Young's modulus increases from 104.407 to 106.765 MPa. The addition of Nano filler improves the polymer's elongation behavior, which ranges from 15.94 MPa for pure PVA polymer film to $239.52 \mathrm{MPa}$ for $1 \%$ loading GO-ZnO hybrid filler. It is well understood from the results that the increase in the percentage of GO-ZnO with PVA is responsible for reinforcing and hardening of the PVA matrix. The thermal stability was confirmed through TGA and DSC analysis. At $600^{\circ} \mathrm{C}$ the \% of weight loss is 94.10 and it decreases to 90.75 with the addition of $5 \%$ of nano particle. The DC conductivity studies of PVA polymer is $9.952 \times 10^{-8} \mathrm{~s} \mathrm{~cm}^{-1}$ and is increased as $5.983 \times 10^{-7} \mathrm{~s} \mathrm{~cm}^{-1}$ with $1 \%$ of nano particle loading and reaches the value $2.895 \times 10^{-6} \mathrm{~s} \mathrm{~cm}^{-1}$ by the incorporation of $5 \% \mathrm{GO}-\mathrm{ZnO}$ nano particle. PVA/GO$\mathrm{ZnO}$ can be regarded as prominent material combining the good conducting properties of graphene oxide and important properties of Zinc Oxide for potential applications in the diverse scientific applications. Graphene as a carbonaceous material has outstanding properties which make it attention-grabbing material. Graphene is conductive in nature and has enormous future role in almost all areas of material science such as paints, varnishes, plastics, foams, and inks, induction heaters, in the construction of materials (concrete, cements, etc.), metallurgical processes, energy storage materials, catalysts, biocompatible implants. $\mathrm{ZnO}$ as a cheap material can be used to improve the mechanical properties of polymer nanocomposites. Fabrication of $\mathrm{ZnO}$ as a UV protection system can make it or $\mathrm{ZnO}$ based nanocomposite a robust candidate especially in polymer industry. Addition of $\mathrm{ZnO}$ nanoparticles into polymers can expand mechanical, electrical and optical properties of polymers because of strong interfacial interaction among organic polymer and inorganic nanoparticles due to its small size, large specific area. Thus nanocomposites of this type can be widely used in coatings, plastics, rubbers and other useful applications. PVA/GO-ZnO based nanoparticles can be applied in construction of LEDs or optoelectronic devices. PVA/GO-ZnO based nanocomposites can also be utilized as biosensors development. Comprehensive study is required on PVA/GO-ZnO based nano materials for their further applications in divergent areas. The chief limitations of PVA/GO-ZnO nanocomposites are post-recovery challenges, reusability, and recyclability. Research is in progress to overcome such limitations. 


\section{References}

1) Roy M, Nelson JK, MacCrone RK, Schadler LS, Reed CW, Keefe R, et al. Polymer nanocomposite dielectrics - the role of the interface. IEEE Transactions on Dielectrics and Electrical Insulation. 2005;12(4):629-643. Available from: https://dx.doi.org/10.1109/tdei.2005.1511089.

2) Starr FW, Schrøder TB, Glotzer SC. Molecular Dynamics Simulation of a Polymer Melt with a Nanoscopic Particle. Macromolecules. 2002;35(11):44814492. Available from: https://dx.doi.org/10.1021/ma010626p.

3) Jancar J, Douglas JF, Starr FW, Kumar SK, Cassagnau P, Lesser AJ, et al. Current issues in research on structure-property relationships in polymer nanocomposites. Polymer. 2010;51(15):3321-3343. Available from: https://dx.doi.org/10.1016/j.polymer.2010.04.074.

4) Lee C, Wei X, Kysar JW, Hone J. Measurement of the Elastic Properties and Intrinsic Strength of Monolayer Graphene. Science. 2008;321(5887):385-388. Available from: https://dx.doi.org/10.1126/science.1157996.

5) Gómez-Navarro C, Burghard M, Kern K. Elastic Properties of Chemically Derived Single Graphene Sheets. Nano Letters. 2008;8(7):2045-2049. Available from: https://dx.doi.org/10.1021/nl801384y.

6) Balandin AA, Ghosh S, Bao W, Calizo I, Teweldebrhan D, Miao F, et al. Superior Thermal Conductivity of Single-Layer Graphene. Nano Letters. 2008;8(3):902-907. Available from: https://dx.doi.org/10.1021/nl0731872.

7) Bolotin KI, Sikes KJ, Jiang Z, Klima M, Fudenberg G, Hone J, et al. Ultrahigh electron mobility in suspended graphene. Solid State Communications. 2008;146:351-355. Available from: https://dx.doi.org/10.1016/j.ssc.2008.02.024.

8) Stoller MD, Park S, Zhu Y, An J, Ruoff RS. Graphene-Based Ultracapacitors. Nano Letters. 2008;8(10):3498-3502. Available from: https://dx.doi.org/10. 1021/nl802558y.

9) Zhang Y, Tan YW, Stormer HL, Kim P. Experimental observation of the quantum Hall effect and Berry's phase in graphene. Nature. 2005;438(7065):201204. Available from: https://dx.doi.org/10.1038/nature04235.

10) Shen J, Hu Y, Shi M, Lu X, Qin C, Li C, et al. Fast and Facile Preparation of Graphene Oxide and Reduced Graphene Oxide Nanoplatelets. Chemistry of Materials. 2009;21(15):3514-3520. Available from: https://dx.doi.org/10.1021/cm901247t.

11) Shen J, Hu Y, Li C, Qin C, Shi M, Ye M. Layer-by-Layer Self-Assembly of Graphene Nanoplatelets. Langmuir. 2009;25(11):6122-6128. Available from: https://dx.doi.org/10.1021/la900126g.

12) Gautam S, Sharma B, Jain P. Structural Applications of Graphene Based Biopolymer Nanocomposites. In: InGraphene Based Biopolymer Nanocomposites. 2021;p. 61-81. Available from: https://doi.org/10.1007/978-981-15-9180-8_4.

13) Huang Y, Ding J, Chen X, Sun X. Synthesis, Mechanical Property, and Thermal Stability of Reduced Graphene Oxide-Zinc Oxide/Cyanate Ester/Bismaleimide Resin Composites. Journal of AdhesionScienceTechnology. 2016;31:1348-1360. Available from: https://doi.org/10.1080/01694243. 2016.1256634 .

14) Ramezanzadeh B, Attar MM. Studying the corrosion resistance and hydrolytic degradation of an epoxy coating containing ZnO nanoparticles. Materials Chemistry and Physics. 2011;130(3):1208-1219. Available from: https://dx.doi.org/10.1016/j.matchemphys.2011.08.065.

15) Kumar SV, Huang NM, Yusoff N, Lim HN. High performance magnetically separable graphene/zinc oxide nanocomposite. Materials Letters. 2013;93:411414. Available from: https://dx.doi.org/10.1016/j.matlet.2012.09.089.

16) Zhong L, Yun K. Graphene Oxide-Modified ZnO Particles: Synthesis, Characterization, and Antibacterial Properties. Int J Nanomed. 2015;10:79-92. Available from: https://doi.org/10.2147/IJN.S88319.

17) Zheng J, Ozisik R, Siegel RW. Disruption of self-assembly and altered mechanical behavior in polyurethane/zinc oxide nanocomposites. Polymer. 2005;46(24):10873-10882. Available from: https://dx.doi.org/10.1016/j.polymer.2005.08.082.

18) Padinhattayil S, Rai KS. Fabrication of Graphene Oxide/Zinc Oxide Hybrid Nanocomposite andAssessment on Structural, Thermal and Optical Characterizations. Asian Journal of Chemistry. 2020;32(4):881-886. Available from: https://dx.doi.org/10.14233/ajchem.2020.22483.

19) DeMerlis CC, Schoneker DR. Review of the oral toxicity of polyvinyl alcohol (PVA). Food and Chemical Toxicology. 2003;41(3):319-326. Available from: https://dx.doi.org/10.1016/s0278-6915(02)00258-2.

20) Butnaru E, Cheaburu CN, Yilmaz O, Pricope GM, Vasile C. Poly(vinyl alcohol)/chitosan/montmorillonite nanocomposites for food packaging applications. High Performance Polymers. 2016;28:1124-1138. Available from: https://dx.doi.org/10.1177/0954008315617231.

21) Pavlidou S, Papaspyrides CD. A review on polymer-layered silicate nanocomposites. Progress in Polymer Science. 2008;33(12):1119-1198. Available from: https://dx.doi.org/10.1016/j.progpolymsci.2008.07.008.

22) Selvi J, Parthasarathy V, Mahalakshmi S, Anbarasan R, Daramola MO, Kumar PS. Optical, electrical, mechanical, and thermal properties and nonisothermal decomposition behavior of poly(vinyl alcohol)-ZnO nanocomposites. Iranian Polymer Journal. 2020;29(5):411-422. Available from: https://dx.doi.org/10.1007/s13726-020-00806-8.

23) Gilman JW, Jackson CL, Morgan AB, Harris R, Manias E, Giannelis EP, et al. Flammability Properties of Polymer-Layered-Silicate Nanocomposites. Polypropylene and Polystyrene Nanocomposites †. Chemistry of Materials. 2000;12(7):1866-1873. Available from: https://dx.doi.org/10.1021/cm0001760.

24) Triantafyllidis KS, LeBaron PC, Park I, Pinnavaia TJ. Epoxy-Clay Fabric Film Composites with Unprecedented Oxygen-Barrier Properties. Chemistry of Materials. 2006;18(18):4393-4398. Available from: https://dx.doi.org/10.1021/cm060825t.

25) Yu Y, Zheng J, Li J, Lu L, Yan J, Zhang L, et al. Applications of two-dimensional materials in food packaging. Trends in Food Science \& Technology. 2021;110:443-457. Available from: https://doi.org/10.1016/j.tifs.2021.02.021.

26) Li D, Müller MB, Gilje S, Kaner RB, Wallace GG. Processable aqueous dispersions of graphene nanosheets. Nature Nanotechnology. 2008;3(2):101-105. Available from: https://dx.doi.org/10.1038/nnano.2007.451.

27) Godovsky D. Biopolymers. PVA Hydrogels Anionic Polymerisation Nanocomposites. Advances in Polymer Science. 2000;153:163-204. Available from: 10.1007/3-540-46414-X.

28) Qian XF, Yin J, Huang JC, Yang YF, Guo XX, Zhu ZK. The preparation and characterization of PVA/Ag2S nanocomposite. MaterChemPhys. 2001;68(13):288-289. Available from: https://doi.org/10.1016/S0254-0584(00)00288-1.

29) Park S, Ruoff RS. Chemical methods for the production of graphenes. Nature Nanotechnology. 2009;4(4):217-224. Available from: https://dx.doi.org/10. 1038/nnano.2009.58.

30) Kumar RV, Elgamiel R, Diamant Y, Gedanken A, Norwig J. Sonochemical Preparation and Characterization of Nanocrystalline Copper Oxide Embedded in Poly(vinyl alcohol) and Its Effect on Crystal Growth of Copper Oxide. Langmuir. 2001;17(5):1406-1410. Available from: https://dx.doi.org/10.1021/ la001331s.

31) Kumar RV, Koltypin Y, Cohen YS, Cohen Y, Aurbach D, Palchik O, et al. Preparation of amorphous magnetite nanoparticles embedded in polyvinyl alcohol using ultrasound radiation. Journal of Materials Chemistry. 2000;10(5):1125-1129. Available from: https://dx.doi.org/10.1039/b000440p. 
32) Strawhecker KE, Manias E. Structure and Properties of Poly(vinyl alcohol)/Na+Montmorillonite Nanocomposites. Chemistry of Materials. 2000;12(10):2943-2949. Available from: https://dx.doi.org/10.1021/cm000506g.

33) Valdes L. Resistivity Measurements on Germanium for Transistors. Proceedings of the IRE. 1954;42(2):420-427. Available from: https://dx.doi.org/10. $1109 /$ jrproc. 1954.274680 .

34) Schuetze AP, Lewis W, Brown C, Geerts WJ. A laboratory on the four-point probe technique. American Journal of Physics. 2004;72(2):149-153. Available from: https://dx.doi.org/10.1119/1.1629085.

35) Rao JK, Raizada A, Ganguly D, Mankad MM, Satayanarayana SV, Madhu GM. Investigation of structural and electrical properties of novel CuO-PVA nanocomposite films. Journal of Materials Science. 2015;50(21):7064-7074. Available from: https://dx.doi.org/10.1007/s10853-015-9261-0.

36) Selvi J, Mahalakshmi S, Parthasarathy V. Synthesis, Structural, Optical, Electrical and Thermal Studies of Poly(vinyl alcohol)/CdO Nanocomposite Films. Journal of Inorganic and Organometallic Polymers and Materials. 2017;27(6):1918-1926. Available from: https://dx.doi.org/10.1007/s10904-017-0662-1.

37) Yang S, Lei P, Shan Y, Zhang D. Preparation and characterization of antibacterial electrospun chitosan/poly (vinyl alcohol)/graphene oxide composite nanofibrous membrane. Applied Surface Science. 2018;435:832-840. Available from: https://dx.doi.org/10.1016/j.apsusc.2017.11.191.

38) Yu YH, Lin CY, Yeh JM, Lin WH. Preparation and properties of poly(vinyl alcohol)clay nanocomposite material. Polymer. 2003;44(12):3553-3560. Available from: 10.1016/S0032-3861(03)00062-4.

39) Shaffer MSP, Windle AH. Fabrication and Characterization of Carbon Nanotube/Poly(vinyl alcohol) Composites. Advanced Materials. 1999;11(11):937941. Available from: https://dx.doi.org/10.1002/(sici)1521-4095(199908)11:11<937::aid-adma937>3.0.co;2-9.

40) Du X, Wang S, Liu Y, Lu M, Wu K, Lu M. Self-assembly of free-standing hybrid film based on graphene and zinc oxide nanoflakes for high-performance supercapacitors. Journal of Solid State Chemistry. 2019;277:441-447. Available from: https://doi.org/10.1016/j.jssc.2019.06.003.

41) Zhang R, Wang Y, Ma D, Ahmed S, Qin W, Liu Y. Effects of ultrasonication duration and graphene oxide and nano-zinc oxide contents on the properties of polyvinyl alcohol nanocomposites. Ultrasonics sonochemistry. 2019;59. Available from: 10.1016/j.ultsonch.2019.104731. 